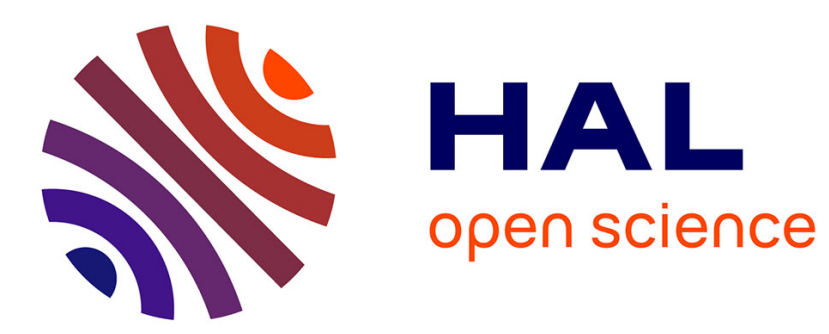

\title{
Analysis of Boundary-Layer Statistical Properties at Dome C, Antarctica
}

Jean-François Rysman, Sébastien Verrier, Alain Lahellec, Christophe Genthon

\section{To cite this version:}

Jean-François Rysman, Sébastien Verrier, Alain Lahellec, Christophe Genthon. Analysis of BoundaryLayer Statistical Properties at Dome C, Antarctica. Boundary-Layer Meteorology, 2015, 156 (1), pp.145-155. 10.1007/s10546-015-0024-x . insu-01143166

\section{HAL Id: insu-01143166 https://hal-insu.archives-ouvertes.fr/insu-01143166}

Submitted on 14 Sep 2015

HAL is a multi-disciplinary open access archive for the deposit and dissemination of scientific research documents, whether they are published or not. The documents may come from teaching and research institutions in France or abroad, or from public or private research centers.
L'archive ouverte pluridisciplinaire HAL, est destinée au dépôt et à la diffusion de documents scientifiques de niveau recherche, publiés ou non, émanant des établissements d'enseignement et de recherche français ou étrangers, des laboratoires publics ou privés. 
Noname manuscript No.

(will be inserted by the editor)

1 Analysis of Boundary Layer Statistical Properties at

2 Dome C, Antarctica

3 Jean-François Rysman • Sébastien

4 Verrier • Alain Lahellec • Christophe

5 Genthon

6 Abstract The boundary layer of the Antarctic Plateau is unique on account

7 of its isolated location and extreme stability. This study investigates the char-

8 acteristics of this boundary layer using wind and temperature measurements

9 from a 45-m high tower located at Dome C. First, spectral analysis reveals

${ }_{10}$ that both fields have a scaling behaviour from 30 minutes to 10 days (spectral

${ }_{11}$ slope $\beta \approx 2$ ). Wind and temperature time series also shows a multifractal

12 behaviour. Therefore, it is possible to fit the moment-scaling function to the

13 universal multifractal model and obtain multifractal parameters for temper-

${ }_{14}$ ature $\left(\alpha \approx 1.51\right.$ and $\left.C_{1} \approx 0.14\right)$ and wind speed $\left(\alpha \approx 1.34\right.$ and $\left.C_{1} \approx 0.13\right)$.

15 The same analysis is repeated separately in winter and summer at six different

16 heights. The $\beta$ parameter shows a strong stratification with height especially in

JF Rysman

UPMC Univ. Paris 06; Université Versailles St-Quentin; CNRS/INSU, LATMOS-IPSL, France E-mail: jfrysman@lmd.polytechnique.fr

$\mathrm{S}$ Verrier

LOCEAN (UPMC/IPSL), CNES, France

A Lahellec

Laboratoire de Météorologie Dynamique, UPMC Univ. Paris 06, France

C Genthon

UJF - Grenoble 1 / CNRS Laboratoire de Glaciologie et Géophysique de l'Environnement (LGGE), France 
17 summer. This means that properties of turbulence change surprisingly rapidly

18 from the ground to the top of the tower.

19 Keywords Boundary Layer - Dome C · Meteorological tower - Scaling ·

$20 \quad$ Statistical properties

\section{${ }_{21} 1$ Introduction}

22 The Antarctic surface consists of a plateau ranging from 2000 to $4000 \mathrm{~m}$ in 23 altitude and covered $98 \%$ by ice (King and Turner, 1997). One of its local 24 maxima is Dome C (3233 m), where the Concordia station has been installed 25 since 1997. At this station, meteorological measurements are taken at the sur26 face with an automated weather station, while daily launched balloons pro${ }_{27}$ vide soundings of the troposphere. As snow surface emissivity is higher than 28 atmosphere emissivity, significant temperature inversion exists in this region 29 at night and during winter (Hudson and Brandt, 2005; Genthon et al., 2013).

30 Moreover, surface winds are weak over the Eastern Antarctic Plateau where

31 the surface is smooth. These features, which inhibit turbulence and vertical 32 motions, explain the extremely stable boundary layer at Dome C. The bound33 ary layer may remain stable for several months almost without interruption, 34 leading to remarkable properties. The analysis of these properties is of high in35 terest to meteorologists since it provides the opportunity to better understand 36 the characteristics of an extremely stable boundary layer in an unperturbed 37 environment and facilitates the development of parameterizations aimed at 38 global and regional models.

39 Overall, boundary layer properties in Antarctica are poorly studied com40 pared with mid-latitude boundary layer properties because of the difficulty in ${ }_{41}$ performing surface measurements. To fill this gap, a tower was installed in 422007 close to the Concordia station. Instruments were set up to measure wind, 
43 temperature, and humidity at six levels along the $45 \mathrm{~m}$ tower. At present, this 44 is the highest tower that performs continuous measurements in Antarctica.

45 As the boundary layer depth in Antarctica is very shallow, the vertical vari46 ability is considerable and, for this reason, a tower is well adapted to study 47 boundary layer characteristics. The continuous measurements along the ver48 tical are particularly interesting when studying the boundary layer temporal 49 evolution and especially when analyzing the transition between the stable and convective boundary layer that occurs on summer days.

In most Antarctic stations, except for the South Pole and Halley stations, 52 in-situ measurements are simply taken at standard meteorological levels (2 53 and $10 \mathrm{~m})$. In addition, some measurement campaigns at high latitude regions 54 have been performed with an instrumented mast (e.g., King and Turner, 1997; 55 Travouillon et al., 2003; Grachev et al., 2005). As a result, long-term, in-situ, 56 and high-quality measurements of the low atmosphere at high latitude are $57 \quad$ scarce and extremely valuable.

The present study is based on wind and temperature observations collected 59 from the tower at Dome C between January and December 2009. The objective 60 was to study the statistical properties of wind and temperature of the Dome $\mathrm{C}$ ${ }_{61}$ boundary layer together with the vertical variability of these properties. The 62 analysis of the boundary layer is often difficult since processes with various 63 spatial and temporal scales occur conjointly. However, statistical properties 64 known as scaling or self-similarity can characterize this complex system with 65 only a few parameters. Experimental measurements have shown that scaling ${ }_{66}$ properties are found in most geophysical fields and are related to atmospheric 67 turbulence, notably wind and temperature (Gage and Nastrom, 1986), cloud 68 radiance (Tessier et al., 1993), and rainfall (Verrier et al., 2011; Rysman et al., $\left.{ }_{69} 2013\right)$ ). As a result, we chose to use this approach in this paper. 
First, we highlight and analyze the scaling behaviour of wind and tem${ }_{71}$ perature fields. In the second part of this analysis, we use the multifractal 72 framework to obtain parameters that describe the fields intrinsic properties. 73 This is the first time that such an innovative analysis is conducted in Antarc74 tica and for such an extremely stable boundary layer. This approach allows us 75 to characterize the full spectrum of signal variability that is not possible with 76 standard approaches.

\section{$77 \quad 2$ Data}

78 The Concordia scientific station is based on a local maxima called Dome C $79\left(75^{\circ} 06^{\prime} \mathrm{S}, 123^{\circ} 20^{\prime} \mathrm{E}, 3233 \mathrm{~m}\right.$ a.s.l. $)$ in the eastern part of the Antarctic 80 Plateau. The nearest coast is located more than $1000 \mathrm{~km}$ away. The local ${ }_{81}$ slope of the Dome is about $5 \times 10^{-4}$ toward the north and $1 \times 10^{-3}$ toward the ${ }_{82}$ east (based on NASA measurements at a 10' resolution). At this latitude, the 83 sun culminates at $38^{\circ}$ on 21 December, and the winter night extends between

${ }_{84}$ April and September.

85 In this study, we used meteorological instruments deployed along a $45 \mathrm{~m}$ 86 tower located $700 \mathrm{~m}$ from the Concordia station. The tower position was chosen ${ }_{87}$ with respect to the atmospheric flow in order to minimize the influence of 88 station buildings. Six Väisälä hygrometers (4 HMP155 and 2 HMP45AC), 89 six pt100 DIN IEC 751 thermistors, and six Young 45106 aero-vanes were 90 mounted at $3.6 \mathrm{~m}, 11 \mathrm{~m}, 18.6 \mathrm{~m}, 25.9 \mathrm{~m}, 33.2 \mathrm{~m}$, and $42.4 \mathrm{~m}$. Measurements ${ }_{91}$ were performed with a 10-second time step and averaged over 30 minutes. 92 Additional technical details can be found in Genthon et al. (2010, 2013). 
$93 \quad 3$ Methodology

$94 \quad 3.1$ Scaling

95 Initially, approaches based on a single exponent, called monofractal approaches,

96 were used to characterize the scaling properties of a field over a given inertial

${ }_{97}$ range. Among these approaches, spectral analysis is widely used. It has been

98 shown that if a physical field presents scaling properties, its power spectral

99 density $E$ (Priestley , 1981), proportional to the square of the modulus of the

100 Fourier transform of the field, follows power-law behaviour as a function of

101 frequency $f$ (i.e., $\log$-log linearity):

$$
E(f) \simeq f^{-\beta}
$$

The $\beta$ value depends on the correlation in a given field: a highly correlated field has a high $\beta$ exponent, while a white noise (uncorrelated field) has a $\beta$ exponent equal to zero (for details, see Rysman et al. (2013)).

Another way to highlight the scaling behaviour of a field is to test the firstorder structure function log-log linearity. The first-order structure function corresponds to the statistical average of the absolute increments for different lags (this is a first-order function analogue to variograms):

$$
S(\delta t)=<|X(t+\delta t)-X(t)|>\simeq \delta t^{H}
$$

where $\delta t$ is the time lag (varying from 0 to the time series length), $S$ the firstorder structure function and $<>$ is the ensemble averaging operator. As for $\beta$, $H$ indicates the smoothness of the field. 
3.2 Multifractal

Subsequently, refinements were proposed in order to take into account the strong inhomogeneity in the energy fluxes (e.g., Yaglom, 1966). These refinements rely on multiplicative cascades, that is, the representation of multiscale variability using a sequence of iterative multiplicative modulations of increasing resolution (e.g., Schertzer and Lovejoy, 1987; She and Leveque, 1994). Such models can usually be parameterized using a few exponents with more or less obvious physical interpretations, thus giving a description of a much wider class of variability than monofractal models (single parameter models). More precisely, multiplicative cascades generate multifractal stochastic fields.

To investigate the validity of these theories with geophysical data, multifractal analysis procedures are applied (e.g., Verrier et al., 2011). The latter relies on the remarkable scaling properties of statistical moments of different orders that generalize the spectral scaling properties to a wider class of process intensities.

Statistically speaking, a field $\Phi$ follows multifractal properties if the statistical moments of the field depend on the resolution in a power-law manner. The power-law exponent only depends on the moment order, so that the statistical moments of the normalized field can be expressed as:

$$
<\Phi_{\lambda}^{q}>\simeq \lambda^{K(q)}
$$

where $<>$ is the ensemble averaging operator, $q$ the moment order (nonnecessarily integer), $\lambda$ the resolution, and $K(q)$ the moment-scaling function, which relates to scaling exponents and moments. In the following, the empirical statistical moments are denoted as $M_{q}(\lambda)$.

Several parameterizations of the fundamental moment-scaling function $K(q)$ exist (e.g., Schertzer and Lovejoy, 1987; She and Leveque, 1994). In this paper, we consider the two-parameter universal form defined by Schertzer and 
Lovejoy (1987). $K(q)$ is thus parametrized by two fundamental parameters, $C_{1}$ (intermittency parameter) and $\alpha$ (index of multifractality):

$$
K(q)=\frac{C_{1}}{\alpha-1}\left(q^{\alpha}-q\right)
$$

where $\alpha \in[0: 2]$ and $\alpha \neq 1$ and $C_{1} \in[0: D]$ with $D$ the dimension of the Euclidean space in which the field is defined (i.e., 1 in the case of time series).

A physical understanding of these parameters allows a given geophysical field to be characterized. $C_{1}$ can be related to the intermittency of the data, that is, the uniformity of the data around the mean. It increases as most of the measured values depart from the mean. $\alpha$ relates to the presence of extreme fluctuations within the field. High values of $\alpha$ indicate a field with a few large singularities (for details on the interpretation of multifractal parameters, see Pecknold et al., 1993; Purdy et al., 2001; Nykanen, 2008).

Often, $\Phi$ cannot be directly related to geophysical fields, because most of these fields and atmospheric processes are better described as low-pass filtered versions of multiplicative cascades. Therefore, a scaling filter such as fractional integration is usually applied (Schertzer and Lovejoy, 1987). Consequently, the properties of the conservative multifractal field $\Phi$ should be distinguished from the (usually) non-conservative integrated fields (the term integrated is related to the fractional integration needed to transform a conservative (non integrated) multifractal field to a realistic physical field (non conservative and integrated)).

The first step of a multifractal analysis is to determine whether the studied field is integrated. To this end, we use spectral analysis: if a field is integrated, its spectral slope is strictly (and notably) greater than 1 . We then use the structure function. Indeed, the $H$ exponent gives the order of fractional integration in the field and physically represents the degree of smoothing involved 


\section{Results}

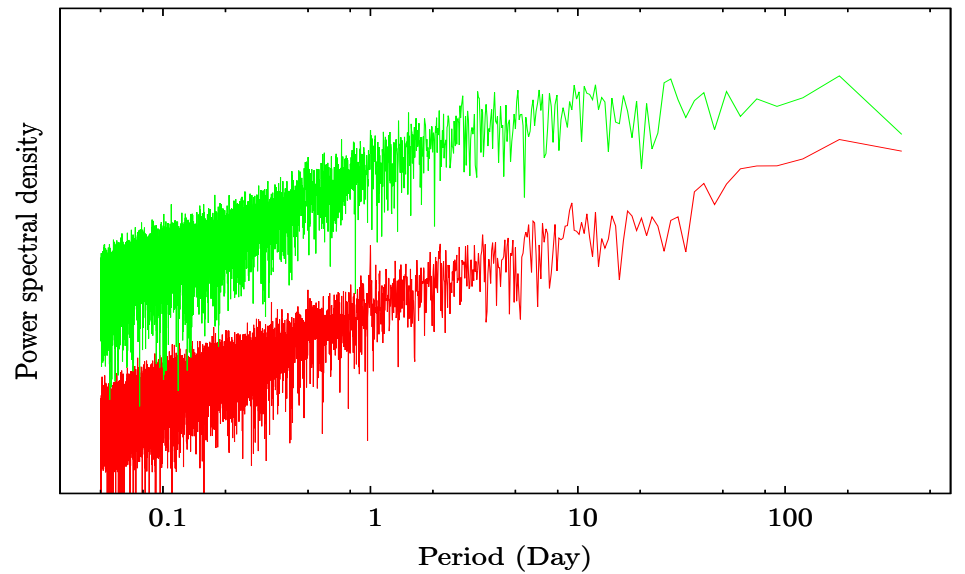

Fig. 1 Power spectral density of temperature $\left(\mathrm{K}^{2} \mathrm{~s}^{-1}\right.$, red line) and zonal wind $\left(\mathrm{m}^{2} \mathrm{~s}^{-3}\right.$, green line) at $42.4 \mathrm{~m}$ in a log-log plot

with the integration. For instance, $H=0$ is associated with a conservative cascade (for details, see de Montera et al., 2009).

Figure 1 reveals that the power spectral density of zonal wind and temperature scale with a slope of respectively 2.20 and 2.02 at $42.4 \mathrm{~m}$ (from 2 hours up to 10 days). In other words, a high temporal autocorrelation exists between these fields, with the temperature at a given time being related to the temperature up to 10 days later. For longer periods of time, both fields appear uncorrelated (spectral slope equals zero) because the meteorological noise is greater than the remaining correlation. The region with periods exceeding 10 days is often called the spectral plateau. The spectral plateau has been highlighted in various meteorological fields in the past (Fraedrich and Larnder, 1993; Olsson, 1995; Fabry, 1996; Lovejoy and Schertzer, 2011; Rysman et al., 2013), with a decorrelation period ranging from 5 days to 1 month, which reveals a similarity among meteorological fields independent of local 
characteristics. It must also be emphasized that both spectra are very similar (similar slope and scaling range), suggesting a relationship between both variables. This similarity can be related to the influence of wind on temperature; for example, when the wind changes direction, local temperature is affected.

Since spectral slopes greater than 1 are notably observed, it means that the fields are integrated. The next step is to determine the degree of fractional integration in the fields. Figure 2 shows the first-order structure functions of the temperature and zonal wind series, averaged over height. Regarding temperature, data only pertains to the period from January to October because temperature sensors were interrupted for a few hours in October. For both temperature and zonal wind, a scaling behaviour is found between 2 and $16 \mathrm{~h}$ with an exponent $H$ of about 0.69 (temperature) and 0.66 (zonal wind). This confirms that for both variables, the observables should perhaps be related to multifractal field only when applying a fractional integration. This is achieved following Lavallée et al. (1993) and de Montera et al. (2009) with the computation of the absolute gradient of the time series.

The empirical moments of the latter are then estimated in order to confirm the validity of Eq. 3. In a log-log plot, the multiscaling behaviour of moments appears as a sequence of straight lines, each associated with a unique moment order. Figure 3 shows the behaviour of statistical moments (between 0 and 2) of temperature and zonal wind as a function of scale in log-log coordinates. Two regimes thus appear: from $2 \mathrm{~h}$ to 2 days and from 2 days to 0.5 month. Red lines at high frequencies show the fit of moment laws in the range $2 \mathrm{~h}-2$ days. In this range of scales, the moments (especially high-order moments) strongly vary with the scale in a way that might be approximated by multifractal laws. Larger scales are characterized by much slighter variations of moments, represented by flat curves at low frequencies. This confirms the findings of the structure function analysis above, wherein a scaling regime was found at 

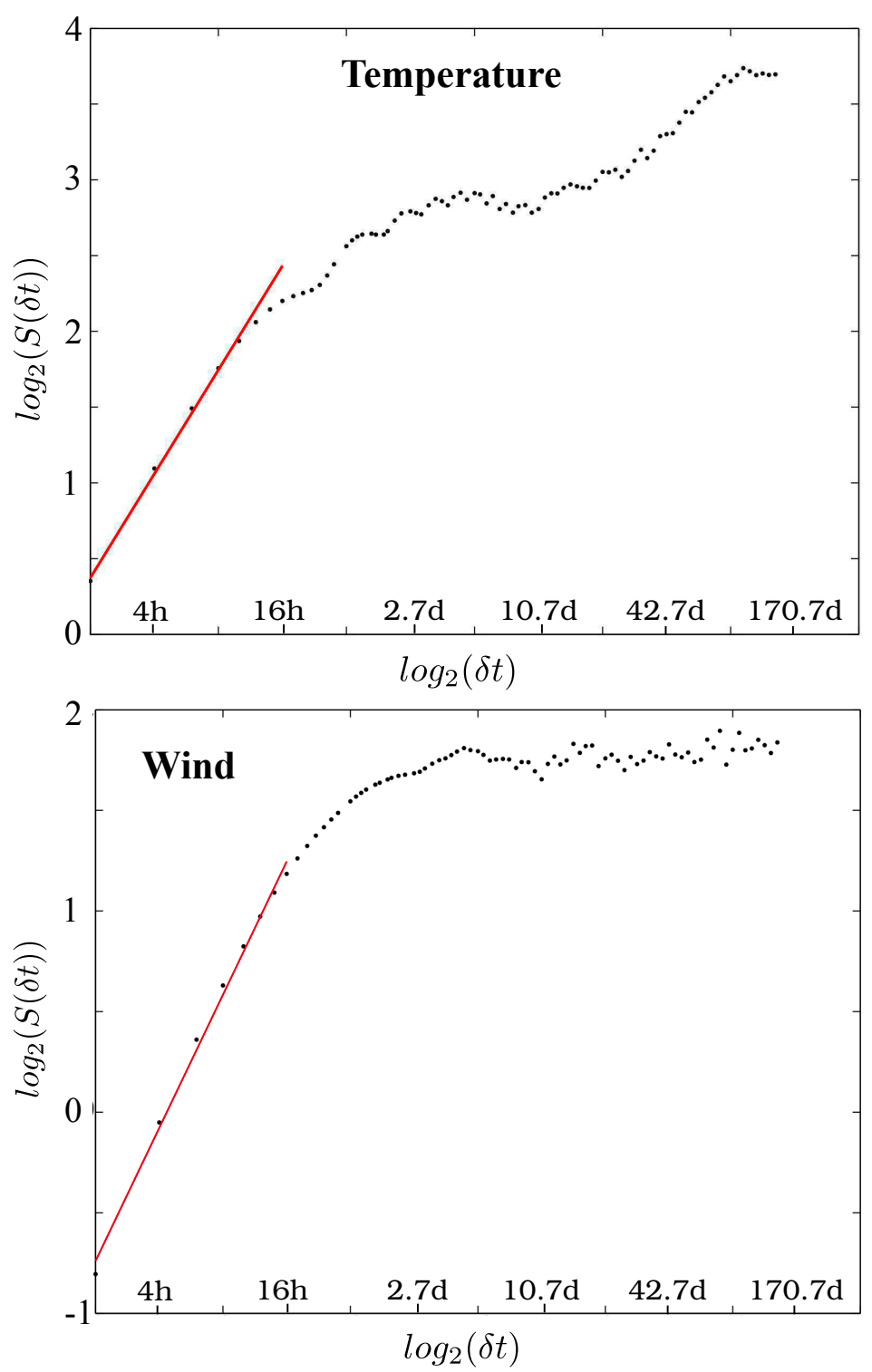

Fig. 2 First-order structure function $(S(\delta t))$ of temperature $(\mathrm{K})$ and zonal wind $(\mathrm{m} . \mathrm{s}-1)$ averaged over heights as a function of time lag $(\delta t)$ ranging from $2 \mathrm{~h}$ to 170.7 days. Linear regressions between 2 and $16 \mathrm{~h}$ are shown as red lines scales. 

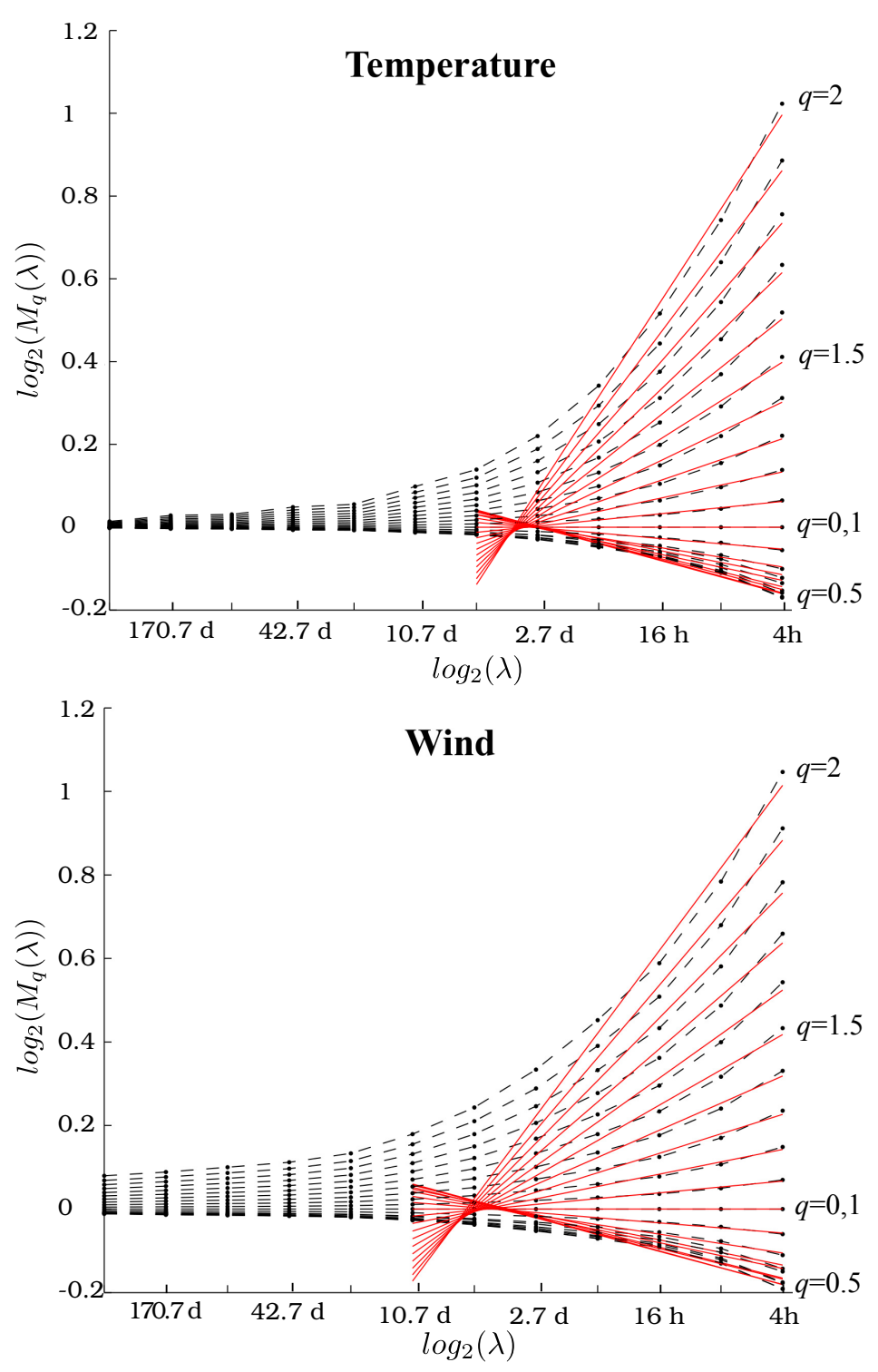

Fig. 3 Empirical moments $\left(M_{q}(\lambda)\right)$ of the absolute temperature and zonal wind temporal gradients, averaged over heights as a function of the resolution ranging from $2 \mathrm{~h}$ to 170.7 days (log-log plot). Each straight line corresponds to a linear regression of the moments of fixed order $q$. The orders taken into consideration are $q=0,0.1,0.2, \ldots, 2.0$

Previous figures showed that zonal wind and temperature fields have multifractal behaviour. The next step focusses on the multifractal parametrization of the $2 \mathrm{~h}-2$ days regime in order to obtain $C_{1}$ and $\alpha$ parameters. Here, the 

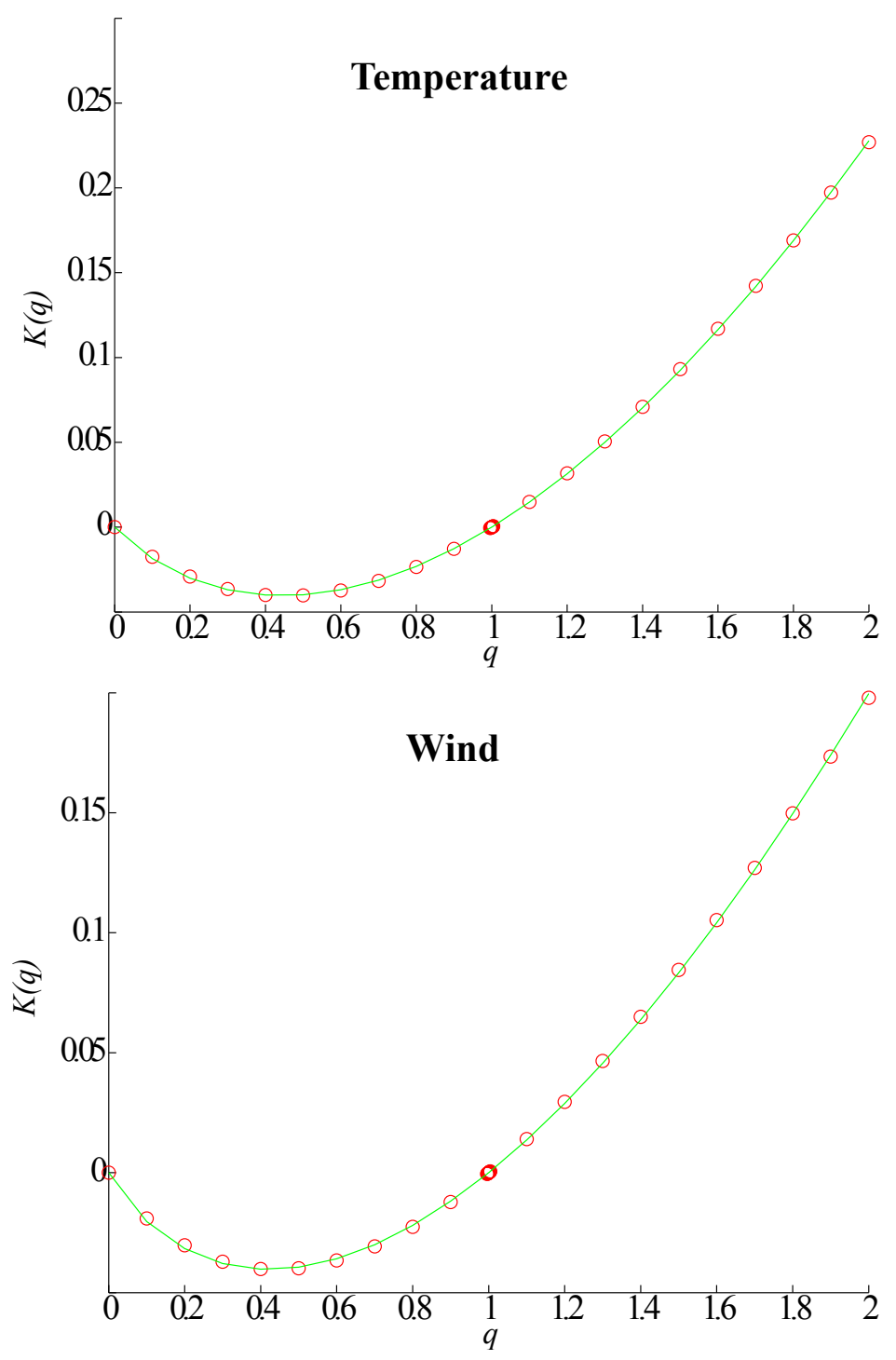

Fig. 4 Empirical moment-scaling function $(K(q))$ (red points), i.e., log-log slopes of the red fit lines in figure 3 and fit with universal multifractal model (green line) between 2 hours and 2 days for temperature and zonal wind averaged over height

slopes of the red fit lines previously computed are represented as a function $K(q)$ for moment order $q$ (Figure 4). The curve of the empirical scaling exponents $K(q)$ is superimposed to the least-square best fit of the universal ( $\alpha$, $\left.C_{1}\right)$ form. First, we observe that the fits are very accurate, thus validating 
the choice of parameterization proposed by Schertzer and Lovejoy (1987). The

best-fit parameters are $\alpha \approx 1.51$ and $C_{1} \approx 0.14$ for temperature and $\alpha \approx 1.34$ and $C_{1} \approx 0.13$ for zonal wind.

These parameters are consistent with previous multifractal analyses. For instance, Schmitt et al. (1992) using laboratory observations obtained $\alpha \approx 1.2$ and $C_{1} \approx 0.15$ for temperature and $\alpha \approx 1.3$ and $C_{1} \approx 0.25$ for wind. Further, Stolle et al. (2009); Lovejoy and Schertzer (2010); Stolle et al. (2012) using model outputs from tropical and mid-latitude regions obtained, in average, for wind and temperature $\alpha \approx 1.8$ and $C_{1} \approx 0.12$. The most significant difference is found for the $\alpha$ parameter, which is lower in our observations compared to the model outputs in tropical and mid-latitude regions. This could indicate that our observations have less extreme values. However, it is difficult to give further interpretation because of the substantial differences between datasets. Additional analysis and measurements are thus required in order to determine whether temperature and wind statistical properties significantly differ at Dome C compared to other parts of the world.

Overall, the monofractal and multifractal results reveal the intrinsic quality of the data. Indeed, positive slopes for spectra and moments highlight the organisation (or correlation) within the geophysical field (see Nykanen, 2008; Rysman et al., 2013), meaning that the noise of data is low compared to the meteorological signal. Moreover, most of the fits show a rather low noise.

Turning to the effect of elevation and season on scaling parameters, the same scaling analysis was applied separately to continuous period of summer (January-February) and winter (July-August) seasons for each height (table $1)$.

Table 1 shows that scaling parameters depend on the season and height. Overall parameters are lower during winter (e.g., for wind $\beta \simeq 2.00 \pm 0.03$ ) than during summer (e.g., for wind $\beta \simeq 2.21 \pm 0.09$ ). Moreover, during winter, 
Table 1 Multifractal parameters as a function of height and season (Summer (JanuaryFebruary) and Winter (July-August)). Missing data are indicated by a - and correspond to periods of interruptions for temperature sensors

\begin{tabular}{|c|c|c|c|c|}
\hline \multirow{3}{*}{ Height (m) } & \multicolumn{4}{|c|}{ Winter } \\
\hline & \multicolumn{2}{|c|}{ Wind } & \multicolumn{2}{|c|}{ Temperature } \\
\hline & $\beta$ & $H$ & $\beta$ & $H$ \\
\hline 3.6 & $2.01 \pm 0.18$ & $0.68 \pm 0.09$ & $2.25 \pm 0.17$ & $0.74 \pm 0.10$ \\
\hline 11 & $1.95 \pm 0.21$ & $0.65 \pm 0.10$ & $2.11 \pm 0.20$ & $0.72 \pm 0.06$ \\
\hline 18.4 & $1.97 \pm 0.22$ & $0.68 \pm 0.13$ & - & - \\
\hline 25.9 & $2.05 \pm 0.17$ & $0.67 \pm 0.11$ & - & - \\
\hline 33.2 & $2.00 \pm 0.14$ & $0.65 \pm 0.10$ & $1.95 \pm 0.24$ & $0.66 \pm 0.09$ \\
\hline \multirow[t]{2}{*}{42.4} & $2.00 \pm 0.23$ & $0.64 \pm 0.11$ & $2.00 \pm 0.22$ & $0.69 \pm 0.09$ \\
\hline & \multicolumn{4}{|c|}{ Summer } \\
\hline 3.6 & $2.06 \pm 0.17$ & $0.68 \pm 0.05$ & $2.14 \pm 0.22$ & $0.91 \pm 0.03$ \\
\hline 11 & $2.14 \pm 0.14$ & $0.72 \pm 0.04$ & $2.05 \pm 0.23$ & $0.85 \pm 0.06$ \\
\hline 18.4 & $2.22 \pm 0.13$ & $0.75 \pm 0.04$ & $1.89 \pm 0.28$ & $0.75 \pm 0.07$ \\
\hline 25.9 & $2.26 \pm 0.12$ & $0.74 \pm 0.04$ & $1.91 \pm 0.17$ & $0.71 \pm 0.06$ \\
\hline 33.2 & $2.28 \pm 0.14$ & $0.75 \pm 0.04$ & $1.92 \pm 0.18$ & $0.69 \pm 0.05$ \\
\hline 42.4 & $2.29 \pm 0.13$ & $0.75 \pm 0.04$ & $1.89 \pm 0.23$ & $0.68 \pm 0.06$ \\
\hline
\end{tabular}

$\beta(2.00 \pm 0.03)$ and $H(0.66 \pm 0.02)$ are rather constant with height for zonal wind while $\beta$ and $H$ decrease from the ground to the top of the tower for temperature (from 2.25 to 2 for $\beta$ and from 0.74 to 0.69 for $H$ ). During summer, the zonal wind shows a stratification with height for the $H$ (from 0.68 to 0.75 ) and the $\beta$ parameters (2.06 to 2.29 ). Regarding temperature, $H$ parameter goes from 0.91 to 0.68 and $\beta$ goes from 2.14 to 1.89 . The $C_{1}$ and $\alpha$ parameters do not seem to be affected by season or height for both fields (not shown in the table). Note that, as the uncertainties in $\beta$ and $H$ values are significant (see table 1), no definitive conclusions can be drawn on the significance of highlighted tendencies. Because boundary layer is almost continuously stable during winter, scaling parameters are characteristics of stable conditions during this season. During summer, boundary layer is alternatively convective and 
stable. Therefore scaling parameters are likely to be affected by both stable and convective conditions during this season.

\section{Summary and Discussion}

This study conducted an analysis of wind and temperature measurements taken at Dome C during the 2009 field campaign. First, the computation of the power spectra of wind and temperature reveals that both fields present scaling properties from 30 minutes to 10 days with a $\beta$ exponent of approximately 2 . Second, the analysis of the first-order structure function provides the degree of fractional integration in both fields (i.e., $H=0.69$ for temperature and $H=0.66$ for zonal wind). The computation of the empirical moment for the temporal gradients of the wind and temperature time series reveals multifractal behaviour. Thus, it is possible to use the universal multifractal model to fit the moment-scaling function $K(q)$ and obtain the $\alpha$ and $C_{1}$ parameters. The same analysis is repeated for winter and summer seasons for six elevations and provides $\beta$ and $H$ parameters in these various conditions. While $\beta$ and $H$ are constant with height during winter for the wind, a stratification of $H$ and $\beta$ parameter is found during summer (e.g., from 2.06 to 2.29 for $\beta$ ). A stratification of $\beta$ and $H$ parameter also exists for the temperature in both winter and summer.

For the first time, this analysis provides scaling parameters in Antarctica for a very stable boundary layer. An important result is the height dependency for $\beta$ and $H$ especially in summer. For the zonal wind the parameters increase from the ground to the top of the tower while for the temperature the parameters decrease from the ground to the top of the tower. This result is very surprising and to the authors best knowledge, this is the first time that such an effect has been observed in these conditions. This behaviour is 
probably related to the different properties of turbulence at the ground and at the top of the tower due to the very strong temperature vertical gradient. In particular, a steeper slope indicates a higher correlation within data; that is turbulence could be stronger close to the ground than at higher levels. Further interpretations require additional measurements of turbulence at Dome C.

This analysis could be used to evaluate parametrizations used in simulations (Stolle et al., 2012, 2009). Indeed, many aspects of a meteorological field can be fully characterized using the multifractal approach with only few coefficients, e.g, the statistical moments and the probability distribution functions of the field for scales ranging from the data resolution to the time series length. Therefore, following the methodology of this paper, simulation outputs (and associated parametrizations) could be evaluated in a new and innovative way. In particular, this method could help to evaluate the statistical relationships between scales in simulations (which is not usually done to our knowledge). Moreover it will help identifying parametrizations that do not respect scalinglaws i.e., that are not physically meaningful.

These computed values and our conclusions must be validated with other measurements obtained in similar conditions, but to our knowledge, no previous scaling (including multifractal) analysis has been conducted in the region. Finally, this analysis also highlights the intrinsic quality of our data. Indeed, most of the fitted functions were found to have relatively little noise with regard to the extreme atmospheric conditions. Since the tower still provides data, it will be possible in the future to improve the accuracy of the scaling parameters. Moreover, measurements from sonic anemo-thermometers recently deployed along the tower will be highly valuable to understand the scaling properties of wind and temperature highlighted in this study. 
Acknowledgements Boundary layer observation and research at Dome $\mathrm{C}$ were supported by the French Polar Institute (IPEV; CALVA program), Institut National des Sciences de IUnivers (Concordia and LEFE-CLAPA programs), and Observatoire des Sciences de IUnivers de Grenoble (OSUG). We are grateful to Yvon Lemaître for his precious help. The authors wish to thank two anonymous reviewers whose valuable feedback greatly improved the manuscript.

\section{References}

de Montera L, Barthès L, Mallet C, Golé P (2009) The effect of rain-no rain intermittency on the estimation of the universal multifractals model parameters. J Hydrometeor 10:493-506, DOI 10.1175/2008JHM1040.1

Fabry F (1996) On the determination of scale ranges for precipitation fields. J Geophys Res 101:12,819-12,826, DOI 10.1029/96JD00718

Fraedrich K, Larnder C (1993) Scaling regimes of composite rainfall time series. Tellus A 45(4):289-298

Gage KS, Nastrom GD (1986) Theoretical interpretation of atmospheric wavenumber spectra of wind and temperature observed by commercial aircraft during GASP. J Atmos Sci 43:729-740, DOI 10.1175/1520-0469

Genthon C, Town MS, Six D, Favier V, Argentini S, Pellegrini A (2010) Meteorological atmospheric boundary layer measurements and ECMWF analyses during summer at Dome C, Antarctica. J Geophys Res Atmos 115:D05104, DOI 10.1029/2009JD012741

Genthon C, Gallée H, Six D, Grigioni P, Pellegrini A (2013) Two years of atmospheric boundary layer observation on a $45-\mathrm{m}$ tower at Dome $\mathrm{C}$ on the Antarctic plateau. J Geophys Res Atmos 118:3218-3232, DOI $10.1002 /$ jgrd. 50128

Grachev AA, Fairall CW, Persson POG, Andreas EL, Guest PS (2005) Stable boundary-layer scaling regimes: the Sheba data. Boundary-Layer Meteorol 
116:201-235, DOI 10.1007/s10546-004-2729-0

Hudson SR, Brandt RE (2005) A Look at the surface-based temperature inversion on the antarctic plateau J Climate 18:1673-1696, DOI 10.1175/JCLI3360.1

King JC, Turner J (1997) Antarctic meteorology and climatology. Cambridge University Press, Cambridge

Lavallée D, Lovejoy S, Schertzer D, Ladoy P (1993) Nonlinear variability of landscape topography: multifractal analysis and simulation. edited by L. DeCola and N. Lam In: Fractals and Geography, Prentice Hall, New Jersey, pp 158-192, 308 pp

Lovejoy S, Schertzer D (2010) Towards a new synthesis for atmospheric dynamics: Space-time cascades. Atmos Res 96:1-52

Lovejoy S, Schertzer D (2011) Space-time cascades and the scaling of ECMWF reanalyses: Fluxes and fields. J Geophys Res Atmos 116:D14117, DOI 10.1029/2011JD015654

Nykanen DK (2008) Linkages between orographic forcing and the scaling properties of convective rainfall in mountainous regions. J Hydrometeor 9:327347, DOI 10.1175/2007JHM839.1

Olsson J (1995) Limits and characteristics of the multifractal behaviour of a high-resolution rainfall time series. Nonlinear Proc Geoph 2:23-29

Pecknold S, Lovejoy S, Schertzer D, Hooge C, Malouin J (1993) The simulation of universal multifractals. edited by J. M. Perdang and A. Lejeune In: Cellular Automata: Prospects in Astronomy and Astrophysics, vol 1, pp 228-267 World Scientific, Hackensack, N. J., 416 pp

Priestley MB (1981) Spectral analysis and time series. Academic Press, New York, $661 \mathrm{pp}$

Purdy JC, Harris D, Austin GL, Seed AW, Gray W (2001) A case study of orographic rainfall processes incorporating multiscaling characterization 
techniques. J Geophys Res 106:7837-7845, DOI 10.1029/2000JD900622

Rysman JF, Verrier S, Lemaître Y, Moreau E (2013) Space-time variability of the rainfall over the western Mediterranean region: A statistical analysis. J Geophys Res Atmos 118:8448-8459, DOI 10.1002/jgrd.50656

Schertzer D, Lovejoy S (1987) Physical modeling and analysis of rain and clouds by anisotropic scaling multiplicative processes. J Geophys Res 92:9693-9714, DOI 10.1029/JD092iD08p09693

Schmitt F, Lovejoy S, Schertzer D, Lavallée D, Hooge C (1992) Estimations directes des indices de multifractals universels dans le champ de vent et de température. C Rendues, de l'Acad Sciences (Paris) 314:749-754

She Z, Leveque E (1994) Universal scaling laws in fully developed turbulence. Phys Rev Lett 72:337-339

Stolle J, Lovejoy S, Schertzer D (2009) The stochastic multiplicative cascade structure of deterministic numerical models of the atmosphere. Nonlinear Proc Geoph 16:607-621

Stolle J, Lovejoy S, Schertzer D (2012) The temporal cascade structure of reanalyses and global circulation models. Q J R Meteorol Soc 138:18951913, DOI 10.1002/qj.1916

Tessier Y, Lovejoy S, Schertzer D (1993) Universal multifractals: theory and observations for rain and clouds Journal of Appl Meteor 32:223-250, DOI $10.1175 / 1520-0450$

Travouillon T, Ashley MCB, Burton MG, Storey JWV, Loewenstein RF (2003) Atmospheric turbulence at the South Pole and its implications for astronomy. Astron Astroph 400:1163-1172, DOI 10.1051/0004-6361:20021814

Verrier S, Mallet C, Barthès L (2011) Multiscaling properties of rain in the time domain, taking into account rain support biases. J Geophys Res Atmos 116:D20119, DOI 10.1029/2011JD015719 
392 Yaglom AM (1966) The influence of fluctuations in energy dissipation on the 393 shape of turbulence characteristics in the inertial interval. Soviet Physics Doklady 11:26-30 